\title{
Métodos quantitativos aplicados à gestão de custos: um estudo descritivo sobre as pesquisas científicas apresentadas nos Congressos Brasileiros de Custos
}

\author{
Quantitative methods applied to management costs: a descriptive study on the \\ scientific data presented in the Brazilian Congress Cost
}

\author{
Carlos Roberto Souza Carmo \\ carlosjj2004@hotmail.com \\ FACIC-UFU
}

\author{
Mônica Aparecida Ferreira \\ monicaapferreira@hotmail.com \\ FACIC-UFU
}

\author{
Vidigal Fernandes Martins \\ vidigalfgv@gmail.com \\ FACIC-UFU
}

\author{
Adeilson Barbosa Soares \\ adeilsonbs@hotmail.com \\ FACIC-UFU
}

\begin{abstract}
Resumo
Após dezessete edições, o Congresso Brasileiro de Custos pode ser visto como um dos principais eventos nacionais de pesquisa e produção científica voltada para área de gestão, controle e análise de custos. O presente trabalho teve por objetivo geral traçar um perfil das publicações científicas voltadas para utilização de métodos quantitativos aplicados na solução de problemas científicos que envolvem custos, apresentadas no Congresso Brasileiro de Custos ao longo do quinquênio 2005-2009. Para tanto, este artigo foi dividido em quatro partes distintas, além da introdução. Na primeira, foram identificadas as justificativas para o seu desenvolvimento, o questionamento direcionador e a metodologia escolhida para respondê-lo. A segunda parte consistiu na elaboração da plataforma teórica sob a qual foi desenvolvida a pesquisa. Na terceira etapa, foram analisados os dados referentes ao perfil dos trabalhos que integraram a área temática voltada para "Aplicação de Modelos Quantitativos na Gestão de Custos", apresentados no evento ao longo do quinquênio 2005-2009. Na última, foram apresentadas as principais considerações sobre a pesquisa, suas limitações e as sugestões para ampliação deste estudo. Ao término desta pesquisa de natureza exploratória e descritiva, foi possível constatar uma predominância na utilização "métodos estatísticos diversos". A "programação linear" foi o segundo método quantitativo mais utilizado dentre os trabalhos que formaram a amostra de pesquisa. Entre outras observações, este trabalho também permitiu constatar a existência de uma predominância de pesquisas que utilizaram algum tipo de estudo de caso real ou a aplicação dos modelos matemáticos em alguma base de aplicação composta por dados reais.

Palavras-chave: Congresso Brasileiro de Custos. Artigos. Métodos Quantitativos.

Abstract:

After seventeen editions, the Brazilian Congress of costs can be seen as a major national event for scientific research focused on the area of management, control and cost analysis. The present work aimed to draw a general profile of scientific publications focused on the use of quantitative methods applied in the solution of scientific problems that involve costs presented in the Brazilian Congress of costs over the five year period 2005-2009. Therefore, this article has been divided into four distinct parts, and the introduction. At first, we
\end{abstract}


identified the reasons for its development, questioning the driver and the methodology chosen to answer it. The second part consisted in developing the theoretical platform which was developed in the research. In the third step, we analyzed the data regarding the profile of the work that made up the thematic area focused on "Application of Quantitative Models in Cost Management," presented at the event over the five year period 2005-2009. At last, the main considerations were presented about the research, its limitations and suggestions for improvement of this study. At the end of this exploratory research and descriptive, we could see a predominance in use "various statistical methods." The "linear programming" was the second most widely used quantitative method among the works that formed the research sample. Among other observations, this work also revealed the existence of a predominance of studies that used some type of real case study or the application of mathematical models based on a composite application with real data.

Keywords: Brazilian Congress of Costs. Articles. Quantitative Methods.

Artigo recebido em: 19.01.2012; Aceito em: 10.07.2012

\section{INTRODUÇÃO}

A pesquisa científica, entendida como "[...] um conjunto de proposições logicamente correlacionadas sobre o comportamento de certos fenômenos que se deseja estudar" (MARCONI e LAKATOS, 2005, p. 80), objetiva fundamentalmente contribuir para a evolução do conhecimento humano.

Esta maneira de entender a pesquisa científica pressupõe que ela seja uma das portadoras do progresso e da racionalidade, constituindo-se, assim, em uma importante peça do contexto social, político e econômico da sociedade em geral.

Ao levar em conta que a pesquisa científica, também, pode ser vista como um dos principais meios de promoção da interatividade entre o conhecimento e sua aplicação, e, ainda, que a sua divulgação em eventos de natureza acadêmica e cientifica permite a disseminação de teorias e práticas voltadas para o contexto organizacional, entre outros, o presente trabalho tem por objetivo geral analisar o perfil das publicações científicas voltadas para utilização de métodos quantitativos aplicados a problemas que envolvem custos, apresentadas no Congresso Brasileiro de Custos, ao longo do quinquênio 2005-2009.

Neste processo de investigação científica, inicialmente, foi avaliada qual evolução da participação destas pesquisas nas cinco edições do congresso escolhido para análise. A seguir, foram identificados quais os principais métodos quantitativos utilizados para solucionar as questões problemas apresentadas nos trabalhos alvo desta pesquisa. Foi analisada, também, qual a natureza destes trabalhos em relação à aplicação prática ou teórica dos métodos quantitativos utilizados.

Para tanto, este trabalho foi dividido em quatro partes distintas, além desta introdução. A primeira, que se inicia no próximo tópico, tratará das justificativas para a escolha da população e da amostra que irão compor a base desta pesquisa. Esta primeira etapa também apresentará o questionamento direcionador da pesquisa e, ainda, a metodologia escolhida para respondê-lo.

A segunda parte do trabalho consistirá na elaboração e apresentação da plataforma teórica sob a qual foi desenvolvida esta investigação. Nesta etapa, serão abordadas as principais metodologias quantitativas utilizadas nos trabalhos que compõem a amostra de pesquisa, no contexto da produção do conhecimento científico na área de custos. 
Ao longo da terceira etapa, serão analisados e discutidos os dados relativos ao perfil dos trabalhos que integraram a área temática voltada para "Aplicação de Modelos Quantitativos na Gestão de Custos", apresentados no Congresso Brasileiro de Custos ao longo do quinquênio 2005-2009.

$\mathrm{Na}$ última parte deste trabalho, serão apresentas as principais considerações sobre todo o processo de pesquisa, as suas limitações e, finalmente, as sugestões para ampliação do estudo realizado.

\section{JUSTIFICATIVAS, PROBleMA DE PESQUiSA E PROCEDIMENTOS METODOLÓGICOS}

Após dezessete edições, desde a realização do primeiro evento em 1994, o Congresso Brasileiro de Custos pode ser visto como um dos principais eventos nacionais de pesquisa e produção científica voltada para área de gestão, controle e análise de custos.

Clemente et al (2010) observam que a Associação Brasileira de Custos, enquanto entidade responsável pela realização daquele congresso, tem contribuído significativamente para a manutenção do conhecimento voltado para a área de custos.

Não só pela importância do evento científico em questão, mas, levando-se em consideração, também, a sua maturidade e, ainda, sua área de especialização, pode-se considerar que os trabalhos publicados no Congresso Brasileiro de Custos representam uma expressiva contribuição para pesquisa científica aplicada a custos. Neste contexto, esta pesquisa tem por objetivo responder o seguinte questionamento direcionador: Qual o perfil das publicações científicas voltadas para utilização de métodos quantitativos aplicados na solução de problemas que envolvem custos, apresentadas no Congresso Brasileiro de Custos ao longo do quinquênio 2005-2009?

Este trabalho científico adotou como população de pesquisa todos os artigos avaliados e publicados nos 16 Congressos Brasileiros de Custos realizados até 2010. Ao considerar que o seu objetivo geral é analisar o perfil das publicações científicas voltadas para utilização de métodos quantitativos aplicados a problemas que envolvem custos, dentro daquela população já identificada, assumir-se-á como amostra o conjunto formado pelos 38 trabalhos aprovados que integraram a área temática voltada para "aplicação de modelos quantitativos na gestão de custos", apresentados no evento, ao longo do quinquênio 2005-2009.

Inicialmente, foi realizada a pesquisa e revisão bibliográfica para construção da plataforma teórica que forneceu o devido suporte à compreensão dos principais métodos quantitativos utilizados nos trabalhos analisados. Sendo que, os seis métodos de análise de dados quantitativos discutidos nesta etapa foram identificados a partir do estudo exploratório realizado com base nas metodologias utilizadas naqueles 38 trabalhos que formaram a amostra de pesquisa do presente trabalho.

Após isso, mediante a pesquisa documental nos anais de todos os congressos que compõem a população de dados pesquisados, procedeu-se à análise descritiva (identificação, evolução e detalhamento) da tipologia dos métodos quantitativos utilizados em pesquisas envolvendo custos e apresentadas ao longo das 16 edições do Congresso Brasileiro de Custos.

A seguir, já contemplando especificamente a amostra de dados escolhidos para realização deste trabalho científico, procurou-se traçar um perfil das publicações científicas voltadas para utilização de métodos quantitativos aplicados na solução de problemas que envolvem custos, apresentadas no Congresso Brasileiro de Custos ao longo do quinquênio 2005-2009. Para tanto, foram identificadas, entre outras, as informações relativas aos seguintes itens: 
- análise evolutiva da participação dos trabalhos publicados, da área de "aplicação de modelos quantitativos na gestão de custos", segundo a população de pesquisa;

- tipologia dos métodos quantitativos utilizados nas pesquisas de custos", segundo a população de pesquisa;

- análise evolutiva relativa da participação das tipologias dos métodos quantitativos utilizados nas pesquisas de custos, segundo a amostra de pesquisa;

- identificação dos tipos de estudos (estudo de caso ou modelo hipotético) utilizados para proposição da aplicação de métodos quantitativos a problemas de custos, segundo a amostra de pesquisa;

- análise evolutiva da participação relativa da tipologia de estudos (estudo de caso ou modelo hipotético) utilizados para proposição da aplicação de métodos quantitativos a problemas de custos, segundo a amostra de pesquisa.

Gil (1999) observa que a pesquisa exploratória é caracterizada por proporcionar uma visão geral acerca de determinado fato real. Neste sentido, devido ao seu objetivo geral, a presente pesquisa pode ser considerada exploratória e descritiva.

\section{PLATAFORMA TEÓRICA}

\subsection{Regressão linear}

A regressão linear pode ser definida como um método que mede a variação média de uma variável, chamada de variável dependente, em relação a uma ou mais variáveis, denominadas de variáveis independentes.

A análise de regressão linear realizada com base em apenas duas variáveis (uma dependente e outra independente) recebe o nome de regressão linear simples. Já a regressão em que se procura explicar o comportamento de uma variável dependente em função de duas ou mais variáveis independentes recebe o nome de regressão linear múltipla.

Ao definir a metodologia de análise do comportamento de variáveis baseado na regressão linear, Stevenson (1986, p. 341) afirma:

\footnotetext{
A regressão linear simples estabelece uma equação matemática linear que descreve o relacionamento entre duas variáveis, uma dependente e outra independente, com a finalidade de estimar valores para uma variável, com base em valores conhecidos da outra.

A regressão linear múltipla envolve três ou mais variáveis, com uma variável dependente e duas ou mais variáveis independentes, com a finalidade de melhorar a capacidade de predição em confronto com a regressão linear simples.
}

Ao descrever processo de execução da análise de regressão, Garrison e Noreen (2001) destacam que esta metodologia de análise consiste em identificar, em um plano cartesiano em que são plotados pontos oriundos de pares ordenados formados pelos valores das variáveis independente (x) e dependente (y), uma linha ou reta capaz minimizar as distâncias entre os pontos encontrados e a média de variação das variáveis analisadas. Ainda segundo Garrison e Noreen (2001), a linha de regressão é obtida por meio da técnica dos mínimos quadrados, que nada mais é do que a minimização da soma dos quadrados das diferenças verticais entre os pontos de dados e a reta de regressão. Essa diferença vertical é denominada resíduo e mede a diferença entre o valor real e o valor obtido para cada observação. 
Anderson et al (2007) observam que a regressão linear simples produz uma linha reta, em um plano cartesiano, a partir de uma função matemática do tipo:

$$
\mathbf{E}(\mathbf{y})=\mathcal{B}_{\mathbf{0}}+\mathcal{B}_{1} \mathbf{x}
$$

Sendo que, ainda conforme Anderson et al (2007, p. 429), os componentes dessa equação podem ser identificados conforme descrito a seguir:

$\mathbf{E}(\mathbf{y})$ = é a média ou valor esperado de y para um determinado valor de $\mathrm{x}$ (variável de estudo);

$\mathcal{B}_{0}=$ é o ponto onde a reta produzida pela equação dada intercepta o eixo y (coeficiente linear ou constante);

$\mathcal{B}_{1}=$ representa o coeficiente de inclinação da reta produzida a partir da equação gerada pela regressão linear;

$\mathbf{x}=$ determina a contribuição da variável dependente X.

Com relação à regressão linear múltipla, Anderson et al (2007) apresentam a seguinte equação para sua definição:

$$
\mathbf{E}(\mathbf{y})=\mathcal{B}_{0}+\mathcal{B}_{1} \mathbf{x}_{1}+\mathcal{B}_{2} \mathbf{x}_{2}+\ldots+\mathcal{B}_{p} \mathbf{x}_{p}
$$

Sendo que, também segundo Anderson et al (2007), os componentes dessa equação podem ser identificados conforme detalhamento a seguir:

$\mathbf{E}(\mathbf{y})$ = é a média ou valor esperado de y (variável de estudo) para um determinado valor de $\mathrm{x}$;

$\mathcal{B}_{0}=$ é o ponto onde a reta produzida pela equação dada intercepta o eixo y (coeficiente linear ou constante);

$\mathcal{B}_{1}=$ representa o efeito que a variável $\mathrm{x}_{1}$ produz sobre $\mathrm{y}$, na reta produzida a partir da equação gerada pela regressão linear;

$\mathcal{B}_{2}=$ representa o efeito que a variável $\mathrm{x}_{2}$ produz sobre $\mathrm{y}$, na reta produzida a partir da equação gerada pela regressão linear;

$\mathcal{B}_{p}=$ representa o efeito que a variável $\mathrm{x}_{\mathrm{p}}$ produz sobre $\mathrm{y}$, na reta produzida a partir da equação gerada pela regressão linear;

$\mathbf{x}=$ determina a contribuição da variável dependente $X$.

Ao analisar a análise de regressão aplicada a custos, Sell (2005, p. 4), observa que esta técnica quantitativa pode ser utilizada para:

- Estimar os valores de uma variável, com base nos valores de outra variável conhecida;

- Explicar os valores de uma variável com base nos valores da outra;

- Predizer futuros valores de uma variável.

\subsection{O método de simulação de Monte Carlo}

O método de Monte Carlo pode ser entendido como uma metodologia de simulação de números aleatórios que permite identificar o grau de probabilidade de um determinado fenômeno de estudo se concretizar ou não, ou melhor, permite identificar propriedades 
predominantes no comportamento de uma variável de estudo a partir da geração e simulação de números aleatórios.

Conforme observado por Prado (2004), existem vários métodos de simulação e um deles é o método de Monte Carlo. Segundo o autor, a aplicação deste método consiste em transformar um conjunto de números aleatórios em outro conjunto de números (variáveis aleatórias), com a mesma distribuição da variável analisada.

Segundo Corrar et al (2004), o processo de simulação de Monte Carlo tem por premissa básica a geração de números aleatórios que atribuem valor às variáveis investigadas e, assim, permitem delinear suas características comportamentais. Corrar et al (2004) ainda observam que os passos básicos para aplicação do método de Monte Carlo podem ser resumidos nas seguintes etapas:

- etapa 1: identificação das distribuições de probabilidades das variáveis aleatórias relevantes para o estudo;

- etapa 2: construção das distribuições de probabilidades acumuladas para cada uma das variáveis definidas no item (a) anterior, quando cabíveis;

- etapa 3: definição dos intervalos de números randômicos (números aleatórios), para cada variável;

- etapa 4: geração dos números aleatórios; e

- etapa 5: simulação dos experimentos.

Mediante um estudo de caso real, Saraiva Júnior et al (2008), observaram que, graças à aplicação do método de simulação de Monte Carlo uma situação problema envolvendo custos, foi possível analisar e tratar, caso a caso, os gastos unitários dos produtos elaborados pela entidade alvo do estudo e, assim, verificar que a adoção de uma média aritmética simples não seria satisfatória.

Em outra situação, também mediante o desenvolvimento de uma pesquisa quantitativa e empírica, Pamplona e Silva (2005) observaram que a utilização da simulação de Monte Carlo, aliada a planilhas eletrônicas, permitiu implementar a tomada de decisões relacionadas a custo, e de caráter imediato, com expressivas agilidade e precisão.

\subsection{Análise envoltória de dados (Data Envelopment Analysis-DEA)}

A metodologia baseada de análise envoltória de dados, doravante denominada apenas de DEA (Data Envelopment Analysis), pode ser entendida como um método que fornece uma medida de desempenho comparativa que leva em consideração o uso de múltiplas alternativas oriundas de hipóteses orientadas por diferentes desempenhos das variáveis analisadas.

Ao abordar esta metodologia de análise Macedo e Cavalcanti (2009) afirmam que a DEA utiliza a programação linear matemática para construir uma fronteira não-paramétrica sobre os dados, onde medidas de eficiência são calculadas em relação a esta fronteira.

Segundo Lins e Meza (2000), a DEA apresenta-se como um método que fornece uma medida de desempenho capaz de comparar a eficiência de várias unidades similares mediante a consideração explícita do uso de suas múltiplas entradas para a produção de múltiplas saídas. Por isso, esta metodologia faz com que a tomada de decisões seja orientada por um único indicador, porém, considerando várias abordagens de desempenho diferentes.

Ao utilizar a DEA para a resolução de problemas de natureza empírica e relacionados a custos e desempenho, Melo et al (2008) prepuseram um modelo de avaliação do desempenho de doze das principais companhias do setor elétrico brasileiro no ano de 2005. Ao final da pesquisa, os autores afirmaram que modelo concebido com base na DEA permitiu conjugar 
indicadores financeiros e não-financeiros como uma alternativa de análise do desempenho organizacional.

Mediante o uso de um exemplo ilustrativo e ao analisarem questões sobre o valor e sobre como os custos influenciam na formação de venda, Macedo e Rosadas (2005) afirmam que uma modelagem baseada na DEA tem "[...] o poder de discriminar as alternativas de custo $\mathrm{x}$ benéfico (valor agregado) em opções atraentes e não atraentes para os clientes, levando em consideração as características técnicas e o preço dos produtos" e, consequentemente, auxiliar os gestores na tomada de decisões relacionadas à formação de preços venda.

\subsection{Matemática financeira aplicada à análise de investimentos}

$\mathrm{Na}$ concepção de modelos de avaliação de empresas, faz-se necessário elaborar séries temporais capazes de prever os fluxos de caixas futuros esperados de um empreendimento. Nesse sentido, Martins (2001, p. 12) observa:

[...] no longo prazo, todos [os modelos de avaliação] são nada mais nada menos do que distribuições temporais diferentes do mesmíssimo fluxo físico de caixa. Assim, no longo prazo, são todos convergentes para um único valor de lucro, com dois únicos fatores a produzir resultados diferentes: a consideração ou não dos efeitos da inflação e a consideração ou não do custo de oportunidade do capital próprio.

Nessa linha de raciocínio, Barreto $(1999$, p. 7) destaca que o valor de uma empresa está diretamente relacionado à sua capacidade de gerar benefícios para os seus proprietários.

Assim, neste tipo de análise, o desafio concentra-se na estimativa dos fluxos a serem considerados no processo da análise de investimentos. Pois, a identificação dos montantes desses fluxos a valor presente na data da avaliação e, ainda, a identificação das taxas de retorno relacionadas ao investimento analisado são basicamente ferramentas utilizadas pela matemática financeira.

\subsection{Programação linear}

Segundo Corrar et al (2004), a programação linear foi criada a partir da Segunda Guerra Mundial com o objetivo de resolver problemas de logística militar. Ainda conforme Corrar et al (2004), sua aplicação efetiva ocorreu em 1947, mediante o desenvolvimento do "método simplex", usado até hoje como base lógica das planilhas eletrônicas.

Ao apresentar uma definição para a programação linear, Prado (1998, p. 15) afirma que ela “[...] permite estabelecer a 'mistura' ótima de diversas variáveis segundo uma função linear de efetividade e satisfazendo a um conjunto de restrições lineares para estas variáveis".

De acordo com Horngren et al (2000), técnicas matemáticas como aquelas empregadas pela programação linear ajudam os tomadores de decisões a resolverem, por exemplo, problemas relacionados à programação da produção de forma a maximizar a margem de contribuição, mediante a redução dos custos variáveis, diante de condições restritivas de produção e recursos.

Sob uma ótica mais técnica e ampla, a programação linear pode ser definida como uma ferramenta que busca a otimização de resultados para problemas relacionados às condições restritivas em que ambos, problema e restrições, podem ser linearizados na forma de funções matemáticas que originam retas, portanto, funções lineares ou, ainda, funções do primeiro grau. 


\subsection{Métodos estatísticos diversos}

A estatística pode ser entendida como um conjunto de metodologias aplicadas na sumarização, interpretação e previsão de fenômenos futuros com no estudo de fenômenos ocorridos no passado. Dentre tais metodologias, destacam-se três grandes grupos. O primeiro deles é o da metodologia estatística descritiva. $\mathrm{O}$ segundo grupo é o das probabilidades. $\mathrm{O}$ último diz respeito às técnicas de estatística inferencial.

A estatística descritiva pode ser entendida como um conjunto de técnicas que tem por objetivo em resumir dados e informações, apresentando-as de forma mais ordenada e simples possível e de acordo com a finalidade pesquisada (BRUNI, 2008). Ou seja, é a ramificação da estatística que utiliza metodologias voltadas para a descrição e sumarização de um conjunto de dados.

Segundo Bruni (2008), a estatística de probabilidades ocupa-se da explicação da freqüência de ocorrência de determinados fenômenos e, com isso, mediante o uso de teorias de probabilidades, estuda o risco de tais eventos se repetirem, ou não, em momentos futuros.

A estatística inferencial, conforme observa Bruni (2008), tem por objetivo compreender o comportamento de um universo de fenômenos pesquisados, mediante o estudos de amostras extraídas deste universo.

Cada um destes ramos da estatística utiliza um conjunto de ferramentas próprias e adequadas aos seus objetivos, conforme exemplos demonstrados no Quadro 1, apresentado a seguir.

Quadro 1: Exemplificação e ramos e "ferramentas" estatísticas

\begin{tabular}{|l|l|}
\hline Ramo estatístico & Exemplos de "ferramentas estatísticas" \\
\hline Estatística descritiva & $\begin{array}{l}\text { média aritmética, média geométrica, média harmônica, média } \\
\text { ponderada, mediana, moda, variância, desvio padrão, coeficiente de } \\
\text { variação, etc.. }\end{array}$ \\
\hline Estatística de probabilidades & $\begin{array}{l}\text { probabilidade normal, probabilidade de poisson, probabilidade } \\
\text { hipergeométrica, probabilidade binomial, estatística "t" de student, } \\
\text { estatística "f", etc.. }\end{array}$ \\
\hline Estatística inferencial & $\begin{array}{l}\text { testes de hipóteses, testes e intervalos de - significância, testes de } \\
\text { hipótese, erro estatístico, teste z, distribuição t de student, } \\
\text { normalização ou distribuição normal, análise de variância, etc. }\end{array}$ \\
\hline
\end{tabular}

Fonte: Adaptado pelos autores com base em Bruni (2008)

Cabe destacar que a metodologia de análise de regressão linear também é um método estatístico do ramo da estatística inferencial. Contudo, a esta metodologia quantitativa foi dedicado um tópico específico logo no início da formulação da presente plataforma teórica, ao invés de abordá-lo neste tópico do trabalho. Justifica-se o tratamento diferenciado prestado à regressão linear em função da quantidade trabalhos da amostra de pesquisa que utilizaram especificamente esta metodologia (8 em um total 38 trabalhos). Adicionalmente, observa-se que a classificação, ou melhor, o grupo "métodos estatísticos diversos" foi identificado a partir de uma grande variedade de ferramentas estatísticas utilizadas, de forma combinada e/ou isoladamente, ao longo da maioria daqueles trabalhos que compuseram a amostra de pesquisa deste trabalho (13 em um total 38 trabalhos).

\section{ANÁLISE DOS DADOS}

Inicialmente, realizou-se um estudo exploratório com base na população de dados desta pesquisa, portanto, nos anais das dezesseis primeiras edições do Congresso Brasileiro de Custos (1994-2010). Nesta etapa inicial, foram identificados, pelo menos, 137 trabalhos 
relacionados aos métodos quantitativos já discutidos no tópico relativo à plataforma teórica da presente pesquisa, conforme resumido pelos dados contidos na Tabela 1.

Tabela 1: Identificação da tipologia dos métodos quantitativos utilizados em pesquisas envolvendo custos, nos artigos apresentados ao longo de todas as 16 edições do Congresso Brasileiro de Custos

\begin{tabular}{l|c}
\hline Método quantitativo & Quantidade de trabalhos \\
\hline Regressão linear & 39 \\
\hline Simulação de Monte Carlo & 13 \\
\hline Análise envoltória de dados (DEA) & 14 \\
\hline Matemática financeira aplicada à análise de investimentos & 5 \\
\hline Programação linear & 17 \\
\hline Métodos estatísticos diversos & 49 \\
\hline Total & $\mathbf{1 3 7}$ \\
\hline
\end{tabular}

Fonte: elaborado pelos autores com base nos dados da pesquisa

Até o XI Congresso Brasileiro de Custos, realizado em 2004, foram apresentados menos de um terço $(44 / 137=32,12 \%)$ daqueles 137 trabalhos que utilizaram um dos 6 métodos quantitativos discutidos no tópico relativo à plataforma teórica desta pesquisa, conforme poderá ser constatado um pouco mais adiante, mediante consulta às informações contidas na Figura 1. Ou seja, independentemente da área temática de classificação nos 11 primeiros Congressos Brasileiros de Custos (1994 até 2004), em média, 4 trabalhos por congresso utilizaram um daqueles métodos quantitativos discutidos nesta pesquisa científica.

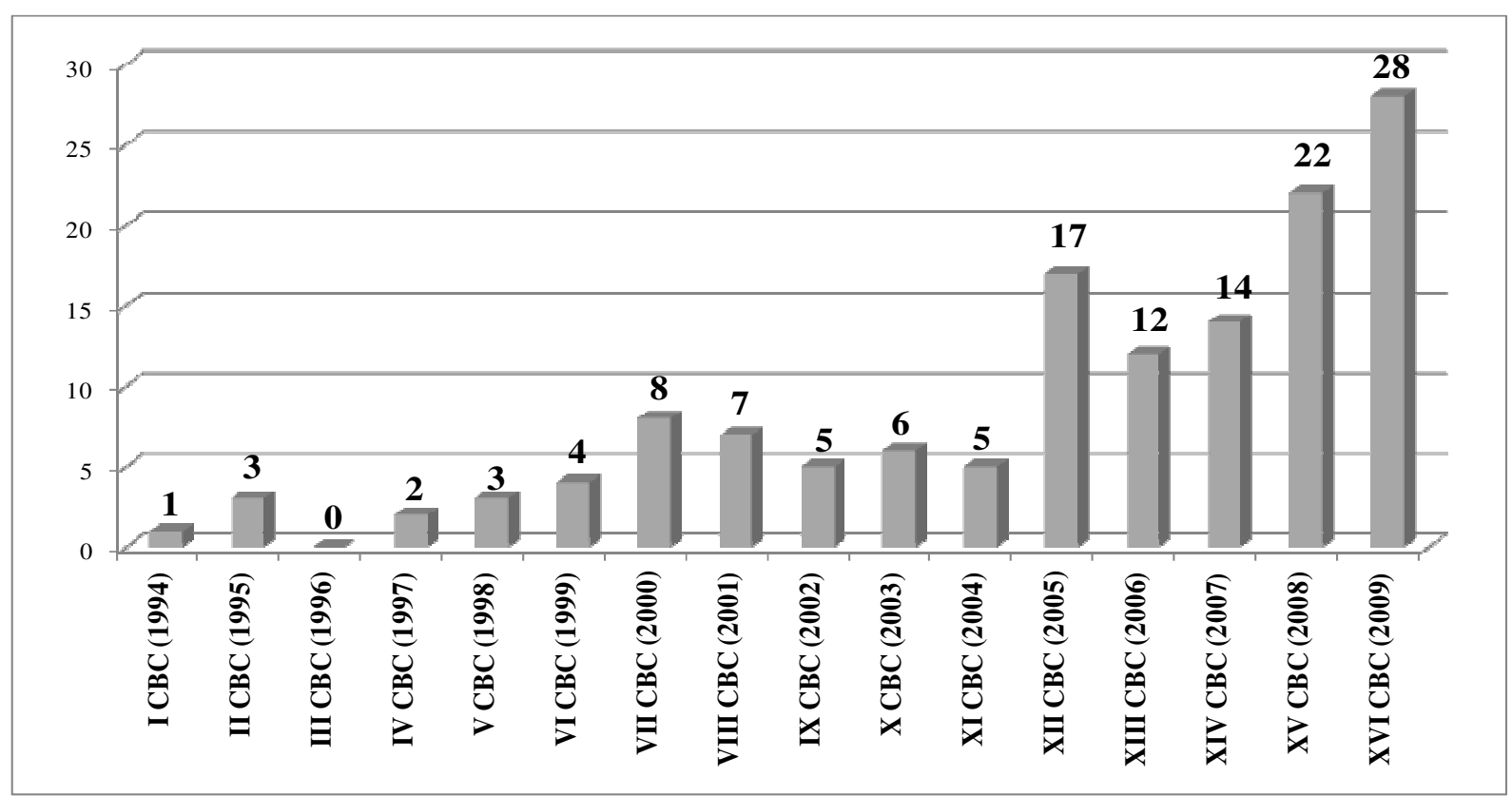

Figura 1: Evolução da tipologia dos métodos quantitativos utilizados em pesquisas envolvendo custos, ao longo de todas as 16 edições do Congresso Brasileiro de Custos Fonte: elaborado pelos autores com base nos dados da pesquisa

A partir de 2005, ocorreu um incremento na média de trabalhos que utilizaram um dos 6 métodos quantitativos discutidos anteriormente. Ou seja, a média de 4 trabalhos por evento 
alcançada no período compreendido entre 1994 e 2004 se elevou para, aproximadamente, 19 trabalhos por evento ao longo do quinquênio 2005-2009.

Em relação aos métodos quantitativos identificados na população de pesquisa, conforme poderá ser visto mais adiante, na Tabela 2, percebe-se uma predominância na utilização "métodos estatísticos diversos" (35,7\%) dentre os trabalhos apresentados ao longo dos 16 anos de realização do Congresso Brasileiro de Custos.

Em segundo lugar, conforme os dados resumidos na Tabela 2, estão os trabalhos que utilizaram métodos quantitativos relativos à regressão linear (28,5\%). Em terceiro lugar, com $12,4 \%$ do total, foram identificados 17 trabalhos que empregaram o método quantitativo da programação linear. A DEA ficou em quarto lugar ao atingir um percentual de $10,2 \%$. E, dentre os métodos quantitativos menos utilizados, destacaram-se os trabalhos que empregaram a simulação de Monte Carlo e a matemática financeira aplicada à análise de investimentos, com 9,5\% e 3,7\%, respectivamente, do total de trabalhos apresentados nos 16 Congressos Brasileiros de Custos analisados.

Ao analisar os trabalhos que compuseram a amostra desta pesquisa, portanto, somente os trabalhos que contemplaram pesquisas científicas voltadas para utilização de modelos quantitativos aplicados na solução de problemas que envolvem custos, apresentados nos Congressos Brasileiros de Custos realizados ao longo do quinquênio 2005-2009, inicialmente, pôde-se perceber que a participação relativa destes trabalhos, em relação aos demais trabalhos apresentados no evento, vem decrescendo. Pois, em 2005, os trabalhos relativos à área temática de "aplicação de modelos quantitativos na gestão de custos" representavam 3,43\% do total dos trabalhos apresentados no evento, entretanto, no último ano escolhido como amostra desta pesquisa (2009) essa participação relativa caiu para menos de 1,6\%, conforme poderá ser constatado mediante análise das informações contidas na Figura 2, apresentada um pouco mais adiante.

Tabela 2: Detalhamento da tipologia dos métodos quantitativos utilizados em pesquisas envolvendo custos, ao longo de todas as 16 edições do Congresso Brasileiro de Custos

\begin{tabular}{|c|c|c|c|c|c|c|c|}
\hline $\begin{array}{r}\text { Método } \\
\text { Quantit. }\end{array}$ & $\begin{array}{l}\text { Regressão } \\
\text { linear }\end{array}$ & $\begin{array}{c}\text { Simulação } \\
\text { de Monte } \\
\text { Carlo }\end{array}$ & $\begin{array}{c}\text { Análise } \\
\text { envoltória } \\
\text { de dados } \\
\text { (DEA) }\end{array}$ & $\begin{array}{l}\text { Matemát. } \\
\text { financeira } \\
\text { aplicada à } \\
\text { análise de } \\
\text { investim. }\end{array}$ & $\begin{array}{c}\text { Program. } \\
\text { linear }\end{array}$ & $\begin{array}{c}\text { Métodos } \\
\text { estatísticos } \\
\text { diversos }\end{array}$ & Total \\
\hline I CBC (1994) & & & & & & 1 & 1 \\
\hline II CBC (1995) & & & & & 1 & 2 & 3 \\
\hline III CBC (1996) & & & & & & & 0 \\
\hline IV CBC (1997) & & & & & & 2 & 2 \\
\hline V CBC (1998) & 1 & 1 & & 1 & & & 3 \\
\hline VI CBC (1999) & & 2 & & & 1 & 1 & 4 \\
\hline VII CBC (2000) & 2 & 1 & & & 3 & 2 & 8 \\
\hline VIII CBC (2001) & 2 & & & 2 & 2 & 1 & 7 \\
\hline IX CBC (2002) & 1 & 1 & & & & 3 & 5 \\
\hline X CBC (2003) & 5 & & 1 & & & & 6 \\
\hline XI CBC (2004) & 1 & 2 & & & 2 & & 5 \\
\hline XII CBC (2005) & 6 & 2 & 2 & & 3 & 4 & 17 \\
\hline XIII CBC (2006) & 4 & & 1 & & 2 & 5 & 12 \\
\hline XIV CBC (2007) & 4 & & 3 & & 1 & 6 & 14 \\
\hline
\end{tabular}




\begin{tabular}{l|c|c|c|c|c|c|c} 
XV CBC (2008) & 6 & 1 & 2 & 1 & 1 & 11 & 22 \\
\hline XVI CBC $(2009)$ & 7 & 3 & 5 & 1 & 1 & 11 & 28 \\
\hline Total & $\mathbf{3 9}$ & $\mathbf{1 3}$ & $\mathbf{1 4}$ & $\mathbf{5}$ & $\mathbf{1 7}$ & $\mathbf{4 9}$ & $\mathbf{1 3 7}$ \\
\hline $\begin{array}{l}\text { Participação } \\
\text { relativa }\end{array}$ & $\mathbf{2 8 , 5 \%}$ & $\mathbf{9 , 5 \%}$ & $\mathbf{1 0 , 2 \%}$ & $\mathbf{3 , 7 \%}$ & $\mathbf{1 2 , 4 \%}$ & $\mathbf{3 5 , 7 \%}$ & $\mathbf{1 0 0 , 0 \%}$ \\
\hline L
\end{tabular}

Legenda:

$\mathrm{CBC}=$ Congresso Brasileiro de Custos

Fonte: elaborado pelos autores com base nos dados da pesquisa

Após observar as informações contidas na Figura 2, exibida um pouco mais a frente, também pode-se perceber que a quantidade total de trabalhos aprovados ao longo quinquênio 20052009 sofreu uma queda expressiva. Ou seja, em 2005, foram apresentadas 350 pesquisas, e, no final do quinquênio (2009), este número caiu para 253 trabalhos. Ao ponderar este fato, torna-se mais evidente ainda a queda na participação relativa dos trabalhos da área temática de "aplicação de modelos quantitativos na gestão de custos", comentada no parágrafo anterior.

Com relação aos 6 grupos de métodos quantitativos identificados e já discutidos na plataforma teórica deste trabalho, a amostra de pesquisa indicou uma predominância na utilização "métodos estatísticos diversos", ou seja, os trabalhos deste grupo (13 trabalhos) representaram 34\% do total dos trabalhos (38) apresentados ao longo do quinquênio 20052009, conforme será demonstrado um pouco mais adiante com o auxílio da Tabela 3.

A "programação linear" foi o segundo método quantitativo mais utilizado dentre os trabalhos que formaram a amostra desta pesquisa. Contudo, deve-se observar que, doravante, a metodologia de "análise de regressão" foi separada em duas categorias, ou seja, em "regressão linear simples" e "regressão linear múltipla", motivo pelo qual ambas foram classificadas como o terceiro método quantitativo mais utilizado. Caso fossem analisadas conjuntamente, ou seja, sob a denominação única de "análise de regressão", este método quantitativo estaria em segundo lugar, portanto, empatado com a "programação linear".

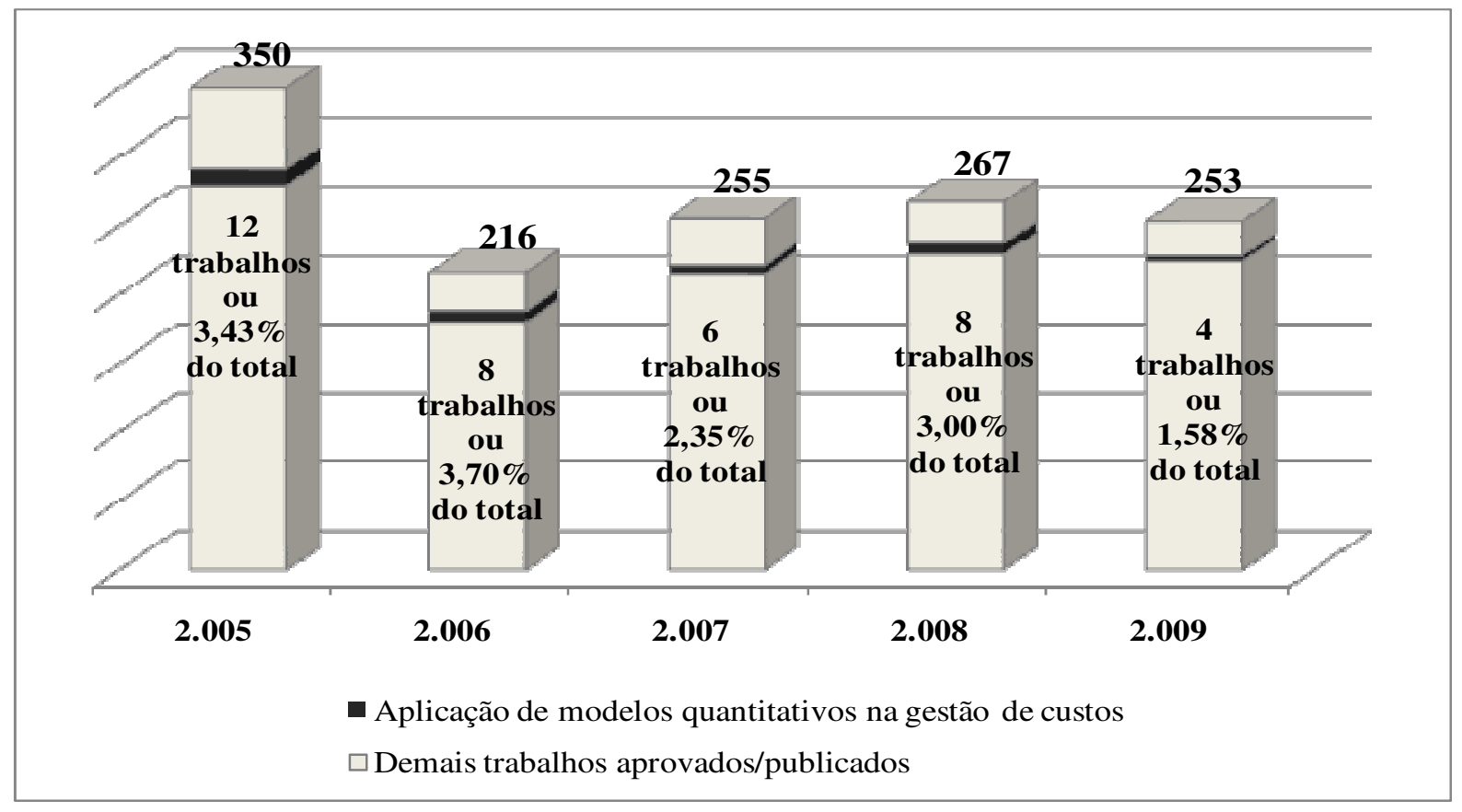


Figura 2: Evolução da participação relativa dos trabalhos da área temática de "aplicação de modelos quantitativos na gestão de custos", apresentados no Congresso Brasileiro de Custos, ao longo do quinquênio 2005-2009

Fonte: elaborado pelos autores com base nos dados da pesquisa

Tabela 3: Detalhamento da tipologia dos métodos quantitativos utilizados em trabalhos da área temática de "aplicação de modelos quantitativos na gestão de custos", apresentados no Congresso Brasileiro de Custos ao longo quinquênio 2005-2009

\begin{tabular}{l|c|c|c|c|c|c|c|c|c|c|c|c}
\hline \multirow{2}{*}{$\begin{array}{l}\text { Tipo de método } \\
\text { quantitativo utilizado }\end{array}$} & $\begin{array}{c}\text { XII CBC } \\
(\mathbf{2 0 0 5})\end{array}$ & $\begin{array}{c}\text { XIII CBC } \\
(\mathbf{2 0 0 6})\end{array}$ & $\begin{array}{c}\text { XIV CBC } \\
(\mathbf{2 0 0 7})\end{array}$ & \multicolumn{2}{c|}{$\begin{array}{c}\text { XV CBC } \\
(\mathbf{2 0 0 8})\end{array}$} & \multicolumn{2}{c|}{$\begin{array}{c}\text { XVI CBC } \\
(\mathbf{2 0 0 9})\end{array}$} & \multicolumn{2}{c}{ Quinquênio } \\
\hline $\begin{array}{l}\text { Regressão linear } \\
\text { simples }\end{array}$ & 2 & $17 \%$ & 2 & $25 \%$ & 0 & $0 \%$ & 0 & $0 \%$ & 0 & $0 \%$ & 4 & $11 \%$ \\
\hline $\begin{array}{l}\text { Regressão linear } \\
\text { múltipla }\end{array}$ & 1 & $8 \%$ & 1 & $13 \%$ & 1 & $17 \%$ & 0 & $0 \%$ & 1 & $25 \%$ & 4 & $11 \%$ \\
\hline $\begin{array}{l}\text { Simulação de Monte } \\
\text { Carlo }\end{array}$ & 2 & $17 \%$ & 0 & $0 \%$ & 0 & $0 \%$ & 1 & $13 \%$ & 0 & $0 \%$ & 3 & $8 \%$ \\
\hline $\begin{array}{l}\text { Análise envoltória } \\
\text { de dados (DEA) }\end{array}$ & 2 & $17 \%$ & 0 & $0 \%$ & 1 & $17 \%$ & 1 & $13 \%$ & 0 & $0 \%$ & 4 & $11 \%$ \\
\hline $\begin{array}{l}\text { Matemát. finan. } \\
\text { aplic. à análise de } \\
\text { invest. }\end{array}$ & 1 & $8 \%$ & 1 & $13 \%$ & 0 & $0 \%$ & 0 & $0 \%$ & 0 & $0 \%$ & 2 & $5 \%$ \\
\hline $\begin{array}{l}\text { Programação linear } \\
\text { Métodos estatísticos }\end{array}$ & 1 & $25 \%$ & 2 & $25 \%$ & 1 & $17 \%$ & 2 & $25 \%$ & 0 & $0 \%$ & 8 & $21 \%$ \\
\hline $\begin{array}{l}\text { Miversos } \\
\text { Total }\end{array}$ & 12 & $100 \%$ & 8 & $25 \%$ & 3 & $50 \%$ & 4 & $50 \%$ & 3 & $75 \%$ & 13 & $34 \%$ \\
\hline $\begin{array}{l}\text { Legenda: } \\
\text { CBC = Congresso Brasileiro de Custos }\end{array}$
\end{tabular}

Fonte: elaborado pelos autores com base nos dados da pesquisa

A "simulação de Monte Carlo" e a "matemática financeira aplicada à análise investimentos" foram os métodos quantitativos menos utilizados segundo a amostra de pesquisa, conforme detalhado na Tabela 3.

Em relação ao contexto em que foram desenvolvidos os trabalhos científicos relativos à área temática de "aplicação de modelos quantitativos na gestão de custos", a amostra pesquisada indicou que, apenas no primeiro ano do quinquênio 2005-2009, portanto, no XII Congresso Brasileiro de Custos, havia uma predominância de trabalhos que utilizavam algum tipo de modelo matemático hipotético ou simulação, conforme será detalhado mais adiante com o auxílio das informações contidas na Tabela 4. Isto é, dos 12 trabalhos apresentados naquele evento, 7 propunham a aplicação de métodos quantitativos com base em exemplos hipotéticos e/ou modelagens quantitativas teóricas. 
Tabela 4: Detalhamento da natureza dos trabalhos da área temática de "aplicação de modelos quantitativos na gestão de custos", apresentados no Congresso Brasileiro de Custos ao longo quinquênio 2005-2009

\begin{tabular}{|c|c|c|c|c|c|c|c|c|c|c|c|c|c|c|}
\hline \multirow{3}{*}{$\begin{array}{l}\text { Tipo de método } \\
\text { quantitativo } \\
\text { utilizado }\end{array}$} & \multicolumn{2}{|c|}{$\begin{array}{c}\text { XII CBC } \\
(2005)\end{array}$} & \multicolumn{2}{|c|}{$\begin{array}{c}\text { XIII CBC } \\
(2006)\end{array}$} & \multicolumn{2}{|c|}{$\begin{array}{c}\text { XIV CBC } \\
(2007)\end{array}$} & \multicolumn{2}{|c|}{$\begin{array}{c}\text { XV CBC } \\
(2008)\end{array}$} & \multicolumn{2}{|c|}{\begin{tabular}{|c|} 
XVI CBC \\
$(2009)$
\end{tabular}} & \multicolumn{4}{|c|}{ Quinquênio } \\
\hline & \multirow{2}{*}{ MHS } & \multirow[t]{2}{*}{ ECR } & \multirow{2}{*}{ MHS } & \multirow{2}{*}{ ECR } & \multirow{2}{*}{ MHS } & \multirow{2}{*}{ ECR } & \multirow{2}{*}{ MHS } & \multirow{2}{*}{ ECR } & \multirow{2}{*}{ MHS } & \multirow{2}{*}{ ECR } & \multicolumn{2}{|c|}{ MHS } & \multicolumn{2}{|c|}{ ECR } \\
\hline & & & & & & & & & & & Qtd. & Part. & Qtd. & Part. \\
\hline $\begin{array}{l}\text { Regressão } \\
\text { linear simples }\end{array}$ & 1 & 1 & & 2 & & & & & & & 1 & $25 \%$ & 3 & $75 \%$ \\
\hline $\begin{array}{l}\text { Regressão } \\
\text { linear múltipla }\end{array}$ & & 1 & & 1 & & 1 & & & & 1 & 0 & $0 \%$ & 4 & $100 \%$ \\
\hline $\begin{array}{l}\text { Simulação de } \\
\text { Monte Carlo }\end{array}$ & 1 & 1 & & & & & & 1 & & & 1 & $33 \%$ & 2 & $67 \%$ \\
\hline $\begin{array}{l}\text { Análise } \\
\text { envoltória de } \\
\text { dados (DEA) }\end{array}$ & 1 & 1 & & & & 1 & & 1 & & & 1 & $25 \%$ & 3 & $75 \%$ \\
\hline $\begin{array}{l}\text { Matemát. finan. } \\
\text { aplic. à análise } \\
\text { de invest. }\end{array}$ & 1 & & & 1 & & & & & & & 1 & $50 \%$ & 1 & $50 \%$ \\
\hline $\begin{array}{l}\text { Programação } \\
\text { linear }\end{array}$ & 3 & & 1 & 1 & 1 & & 1 & 1 & & & 6 & $75 \%$ & 2 & $25 \%$ \\
\hline $\begin{array}{l}\text { Métodos } \\
\text { estatísticos } \\
\text { diversos }\end{array}$ & & 1 & 1 & 1 & 1 & 2 & 1 & 3 & & 3 & 3 & $23 \%$ & 10 & $77 \%$ \\
\hline Total & 7 & 5 & 2 & 6 & 2 & 4 & 2 & 6 & 0 & 4 & 13 & $34 \%$ & 25 & $66 \%$ \\
\hline $\begin{array}{l}\text { Legenda: } \\
\text { MHS= Modelo hip } \\
\text { ECR = Estudo de c }\end{array}$ & caso r & al ou & & $\mathrm{em}$ & 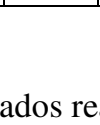 & & & & & & & & & \\
\hline
\end{tabular}

Fonte: elaborado pelos autores com base nos dados da pesquisa

A partir da realização do XIII até o XVI Congresso Brasileiro de Custos, aquela situação observada inicialmente (no XII CBC) se inverteu. Ou seja, a maioria dos trabalhos aprovados na área temática de "aplicação de modelos quantitativos na gestão de custos" passou a utilizar algum tipo de estudo de caso real ou demonstrava a aplicação dos modelos matemáticos pesquisados utilizando alguma base de aplicação composta por dados reais, conforme já demonstrado com o auxílio da Tabela 4.

Ainda com base nos dados apresentados pela Tabela 4, pode-se observar que no período escolhido para análise, portanto, entre os anos de 2005 a 2009, temos, respectivamente, 5, 6, 4, 6 e 4 estudos de caso real ou aplicação em dados reais. Logo, observam-se indícios de uma tendência de estabilização na utilização deste tipo de estudo.

Por fim, ainda com relação à utilização de algum tipo de estudo de caso real ou aplicação dos modelos matemáticos pesquisados utilizando uma base composta por dados reais, destaca-se que, exceto pelos métodos quantitativos da "matemática financeira aplicada a analise de investimentos" e da "programação linear", em todos os outros casos detectou-se uma predominância dos trabalhos de natureza empírica.

\section{CONSIDERAÇÕES FINAIS}

Após dezessete anos desde a realização do primeiro evento em 1994, o Congresso Brasileiro de Custos pode ser visto como um dos principais eventos nacionais de pesquisa e produção científica voltada para área de gestão, controle e análise de custos. 
Devido à sua importância e tradição já consolidadas e, ainda, levando-se em conta o seu alto nível de especialização e concentração na área de custos, deve-se considerar a expressiva contribuição deste evento para pesquisa científica neste segmento ao longo de todos esses anos.

Com o objetivo de analisar o perfil das publicações científicas voltadas para utilização de métodos quantitativos aplicados a custos, dentre todo o universo de trabalhos publicados no Congresso Brasileiro de Custos ao longo das suas 16 edições, este trabalho científico tomou como amostra o conjunto formado pelos 38 trabalhos aprovados no quinquênio 2005-2009 e que integraram a área temática voltada para "aplicação de modelos quantitativos na gestão de custos".

Inicialmente, pôde-se perceber que, mesmo diante da queda no número total de trabalhos aprovados e divulgados no evento, a participação relativa dos trabalhos da área temática escolhida para esta pesquisa decresceu de 2005 (3,43\%) até 2009 (1,58\%). Ou seja, se quantidade de trabalhos voltados para área temática de "aplicação de modelos quantitativos na gestão de custos" se mantivesse estável, em função da queda na quantidade total de trabalhos aprovados e apresentados no quinquênio 2005-2009, aquela participação relativa poderia se elevar.

Comparativamente à quantidade total de trabalhos que utilizou métodos quantitativos em todas as áreas temáticas e em todas as edições do Congresso Brasileiro de Custos (veja informações já apresentadas na Figura 1), os trabalhos específicos da área temática de "aplicação de modelos quantitativos na gestão de custos" tiveram um comportamento inverso. Pois, o número de pesquisas em geral que utilizaram métodos quantitativos aumentou ao longo das 16 edições do Congresso Brasileiro de Custos e, também, no quinquênio 20052009, contudo, o número de trabalhos específicos da área temática de "aplicação de modelos quantitativos na gestão de custos" sofreu um decréscimo, conforme demonstrado ao longo desta pesquisa.

Após analisada toda a amostra da pesquisa, foram identificados 7 grupos distintos de métodos quantitativos utilizados naqueles 38 trabalhos aprovados e publicados nos anais do Congresso Brasileiro de Custos em todo o quinquênio 2005-2009. Ou seja: (i) regressão linear simples, (ii) regressão linear múltipla, (iii) simulação de Monte Carlo, (iv) análise envoltória de dados (DEA), (v) matemática financeira aplicada à análise de investimentos, (vi) programação linear, e, (vii) métodos estatísticos diversos.

A pesquisa indicou uma predominância na utilização "métodos estatísticos diversos", ou seja, os trabalhos deste grupo (13 trabalhos) representaram 34\% do total dos trabalhos (38) apresentados ao longo do quinquênio 2005-2009. A "programação linear" foi o segundo método quantitativo mais utilizado dentre os trabalhos que formaram a amostra desta pesquisa. A "análise de regressão simples" e "regressão múltipla", se analisadas separadamente, ocuparam o terceiro lugar dentre os métodos quantitativos mais utilizados. Contudo, se estas duas categorias fossem agrupadas sob a denominação única de "análise de regressão" e, portanto, fossem analisadas conjuntamente, este método quantitativo estaria em segundo lugar, empatado com a "programação linear". A "simulação de Monte Carlo" e a "matemática financeira aplicada à análise investimentos" foram os métodos quantitativos menos utilizados segundo a amostra de pesquisa.

Em relação ao contexto em que foram desenvolvidos os trabalhos científicos relativos à área temática de "aplicação de modelos quantitativos na gestão de custos", a amostra pesquisada indicou que, apenas no primeiro ano do quinquênio 2005-2009, portanto, no XII Congresso Brasileiro de Custos, havia uma predominância de trabalhos que utilizavam algum tipo de modelo matemático hipotético ou simulação, comparativamente às pesquisas que 
desenvolviam a aplicação de métodos quantitativos mediante a adoção de estudos de casos reais ou aplicações com base em dados reais.

Nos quatro eventos posteriores, portanto, desde a realização do XIII até o XVI Congresso Brasileiro de Custos, a maioria dos trabalhos aprovados na área temática de "aplicação de modelos quantitativos na gestão de custos" utilizaram algum tipo de estudo de caso real ou a aplicação dos modelos matemáticos em alguma base de aplicação composta por dados reais. A esse respeito, também foi possível observar, com base no período compreendido entre os anos 2005 e 2009, a existência de uma tendência de estabilização na quantidade de estudos de natureza empírica voltados para área temática de "aplicação de modelos quantitativos na gestão de custos".

No tocante às limitações do estudo, destaca-se que as evidências detectadas e apresentadas por esta pesquisa, apesar de relevantes, não podem ser generalizadas para toda a produção científica realizada na área de custos como um todo, pois, foram analisados somente os trabalhos que integraram a área temática de "aplicação de modelos quantitativos na gestão de custos" aprovados e publicados nos anais do Congresso Brasileiro de Custos em todo o quinquênio 2005-2009. Por isso, sugere-se realização de um trabalho desta mesma natureza, porém, aplicado a artigos publicados em periódicos internacionais com alto fator de impacto, como forma de comparação a um parâmetro externo. Isto faria com que o presente trabalho migrasse da categoria descritiva para a categoria qualitativa, cujo principal benefício seria uma possível evidenciação do que a pesquisa científica brasileira baseada em métodos quantitativos aplicados a custos está ou não fazendo e, ainda, como ela vem se desenvolvendo com o passar dos anos.

\section{REFERENCIAS}

ANDERSON, David R. et al. Estatística aplicada à administração e economia. 2. ed. São Paulo: Cengage Learning, 2007.

BARRETO, A. C. P. M.. Avaliação econômica financeira de marcas: um estudo de caso sob a perspectiva do modelo de fluxo de caixa descontado. Dissertação de Mestrado em Administração, Faculdade de Economia, Administração e Contabilidade. , Universidade de São Paulo: São Paulo, 1999.

BRUNI, A. L.. Estatística aplicada à gestão empresarial. 2.ed. São Paulo: Atlas, 2008.

CLEMENTE, Ademir et al. Utilização de métodos quantitativos em pesquisa científica: o caso da Associação Brasileira de Custos. ABCustos - Revista da Associação Brasileira de Custos. São Leopoldo, v. 5, n. 2, p.45-61, mai.-ago./2010. Disponível em: <http://www.unisinos.br/abcustos/_pdf/192.pdf> Acesso em: 10 jan. 2011.

CORRAR, Luiz J. et al. Pesquisa operacional para decisão em contabilidade e administração: contabilometria. São Paulo: Atlas, 2004.

GARRISON, Ray H.; NOREEN, Eric W. Contabilidade gerencial. 9. ed. Rio de Janeiro: LTC, 2001.

GIL, A. C. Métodos e técnicas de pesquisa social. São Paulo: Atlas, 1999. 
HORNGREN, Charles T. et al. Contabilidade de custos. 9. ed. Rio de Janeiro: LTC, 2000.

LINS, M. P. E.; MEZA, L. A. Análise envoltória de dados e perspectiva de integração no ambiente de apoio à decisão. Rio de Janeiro: Editora da COPPE/UFRJ, 2000.

MACEDO, Marcelo Álvaro da Silva. ROSADAS, Leandro Azevedo da Silva. Formação do preço de venda: uma proposta de modelagem através do uso de Análise Envoltória de Dados (DEA). In: CONGRESSO BRASILEIRO DE CUSTOS, 12, 2005, Florianópolis. Anais... Florianópolis: Associação Brasileira de Custos, 2005. Disponível em: < http://www.abcustos.org.br/texto/viewpublic?ID_TEXTO=437 >. Acesso em: 22 jan. 2011.

CAVALCANTE, Glaydson Teixeira. Desempenho contábil-financeiro de seguradoras no Brasil: um estudo apoiado em Análise Envoltória de Dados (DEA) para o ano de 2007. In: CONGRESSO BRASILEIRO DE CUSTOS, 16, 2009, Fortaleza. Anais... Fortaleza: Associação Brasileira de Custos, 2009. Disponível em:

$<$ http://www.abcustos.org.br/texto/viewpublic?ID_TEXTO=2863 >. Acesso em: 20 jan. 2011.

MARCONI, Marina de Andrade; LAKATOS, Eva Maria. Fundamentos de metodologia científica. 6. ed. São Paulo: Atlas, 2005.

MARTINS, E. (Org.). Avaliação de empresas: da mensuração contábil à econômica, São Paulo: Atlas, 2001.

MELO, Clayton Levy Lima de, et al. Avaliação do desempenho organizacional: um estudo das companhias do setor elétrico brasileiro com base na Análise Envoltória de Dados (DEA). In: CONGRESSO BRASILEIRO DE CUSTOS, 15, 2008, Curitiba. Anais... Curitiba: Associação Brasileira de Custos, 2009. Disponível em: < http://www.abcustos.org.br/texto/viewpublic?ID_TEXTO=2642 >. Acesso em: 31 jan. 2011.

PAMPLONA, Edson de Oliveira. SILVA, Wander Fonseca da. Contribuição da simulação de Monte Carlo na projeção de cenários para gestão de custos na área de laticínios. In:

CONGRESSO BRASILEIRO DE CUSTOS, 9., 2005, Florianópolis. Anais... Florianópolis: Associação Brasileira de Custos, 2005. Disponível em: < http://www.abcustos.org.br/texto/viewpublic?ID_TEXTO=494 >. Acesso em: 20 jan. 2011.

PRADO, Darci. Teoria das filas e da simulação. 2. ed. Nova Lima: INDG, 2004. v.2.

Programação linear. Belo Horizonte: DG, 1998.

SARAIVA JÚNIOR, Abraão Freires et al. Determinação da margem de contribuição de produtos com variabilidade de preços e custos diretos unitários. In: CONGRESSO

BRASILEIRO DE CUSTOS, 15., 2008, Curitiba. Anais... Curitiba: Associação Brasileira de Custos, 2008. Disponível em:

<http://www.abcustos.org.br/texto/viewpublic?ID_TEXTO=2579>. Acesso em: 20 jan. 2011.

SELL, Isair. Utilização da regressão linear como ferramenta de decisão na gestão de custos. In: CONGRESSO BRASILEIRO DE CUSTOS, 12., 2005, Florianópolis. Anais... 
Florianópolis: Associação Brasileira de Custos, 2005. Disponível em:

<http://www.abcustos.org.br/texto/viewpublic?ID_TEXTO=581>. Acesso em: 10 jan. 2008.

STEVENSON, W. J.. Estatística aplicada à administração. São Paulo: Harbra, 1986. 